Noname manuscript No.

(will be inserted by the editor)

\title{
Polynomial Root Radius Optimization with Affine Constraints
}

\author{
Julia Eaton · Sara Grundel • Mert \\ Gürbüzbalaban · Michael L. Overton
}

Received: date / Accepted: date

\begin{abstract}
The root radius of a polynomial is the maximum of the moduli of its roots (zeros). We consider the following optimization problem: minimize the root radius over monic polynomials of degree $n$, with either real or complex coefficients, subject to $k$ linearly independent affine constraints on the coefficients. We show that there always exists an optimal polynomial with at most $k-1$ inactive roots, that is, roots whose moduli are strictly less than the optimal root radius. We illustrate our results using some examples arising in feedback control.
\end{abstract}

Keywords polynomial optimization · nonsmooth optimization · frequency domain stabilization

\section{Mathematics Subject Classification (2010)}

J. Eaton is supported in part by the NSF-AWM Mentoring Travel Grant and the University of Washington, Tacoma, School of Interdisciplinary Arts \& Sciences Research Fund. M.L. Overton is supported in part by National Science Foundation grant DMS-1317205.

J. Eaton (corresponding author)

School of Interdisciplinary Arts and Sciences, University of Washington Tacoma

Tacoma, WA 98402 USA

Tel.: +1(253) 692.4457

Fax: +1(253) 692.5718

E-mail: jreaton@uw.edu

S. Grundel

Max Planck Institute for Dynamics of Complex Technical Systems

39106 Magdeburg, Germany

M. Gürbüzbalaban

Laboratory for Information and Decision Systems, Massachusetts Institute of Technology Boston, MA 02139 USA

M.L. Overton

Courant Institute of Mathematical Sciences, New York University

New York, NY 10012 USA 


\section{Introduction}

The root radius of a polynomial is the maximum of the moduli of its roots (zeros). This is a fundamental function that arises in many applications. One of the most important application areas is feedback control in the frequency domain, which is modeled by rational system transfer functions. For a transfer function to be discrete-time stable, its denominator - a polynomial - must have its roots in the open unit disk, or equivalently, its root radius must be less than one. Stabilization problems, which require identification of a stable polynomial whose coefficients satisfy certain requirements, have been studied for decades; several of these were mentioned in [2] as being among the "major open problems in control systems theory."

When the coefficients of a polynomial family depend on parameters, it is natural to consider minimizing the polynomial root radius to obtain or enhance stability. However, such an optimization problem is nontrivial because the root radius is not convex, and furthermore it is not locally Lipschitz at points in polynomial space with multiple roots - more specifically, multiple active roots, that is, roots whose moduli equal the maximum modulus. Nonetheless, as we show in this paper, the global minimizers of these optimization problems have a remarkable structural property when dependence on the parameters is affine, or, equivalently, affine constraints are imposed on the polynomial coefficients. Specifically, for the polynomial root radius optimization problem over monic polynomials of degree $n$ with either real or complex coefficients, if $k$ linearly independent affine constraints are imposed on the coefficients, then there always exists an optimal polynomial with at most $k-1$ inactive roots, that is, with at least $n-k+1$ active roots.

As far as we know, such results have not been obtained or even contemplated previously, except that such a conjecture was made, based on experimental results, by Blondel, Gürbüzbalaban, Overton and Megretski [3, Section V]. That paper, building on results of Chen in [11] and [12], obtained stronger results for the special case $k=1$, concerning not only root activity, but also root multiplicity. Specifically, in the case of minimizing the root radius over polynomials with complex coefficients subject to just one affine constraint, it was shown in [3] that there always exists an optimal polynomial of the form $p(z)=(z-\gamma)^{n}$ for some $\gamma \in \mathbb{C}$, meaning not only that there are $n$ active roots, but that they actually coincide. When the coefficients are restricted to be real, it was shown that there always exists an optimal polynomial of the form $p(z)=(z-\gamma)^{m}(z+\gamma)^{n-m}$ for some $\gamma \in \mathbb{R}$ and $m \in\{0, \ldots, n\}$, implying the existence of an optimal polynomial with multiple roots when $n \geq 3$. In contrast, when $k>1$, there may not exist an optimal polynomial with multiple roots.

As a concrete example, consider minimizing the root radius of the cubic polynomial $p(z)=z^{3}+a z+1$ over $a \in \mathbb{R}$, or equivalently, imposing $k=2$ affine constraints: the quadratic coefficient is 0 and the constant coefficient is 1 . The minimal value 1 is attained by the unique optimal polynomial $p_{*}(z)=z^{3}+1$ with three active roots, one more than required by our general theorem, but 
these are the three cube roots of unity and hence are distinct; multiple active roots do not occur. In contrast, consider minimizing the root radius of the polynomial $p(z)=z^{3}+b z^{2}+a z+1$ over $a \in \mathbb{R}, b \in \mathbb{R}$, allowing both the linear and quadratic coefficients to vary, or equivalently, imposing just one constraint: the constant coefficient is 1 . Then the minimal value remains 1 , but the optimal polynomial is no longer unique; any polynomial with $a=b \in[-1,3]$ has root radius 1 , and in particular, when $a=b=-1$, there are two active roots at 1 and one at -1 , while for $a=b=3$, all three active roots coincide at -1 .

The proofs of the results given in [3] for a single constraint were completely different for the cases of real and complex coefficients, with the latter being far more complicated than the former. Our proof of root activity when there are several affine constraints uses a more general approach that applies to both real and complex coefficients and recovers the activity (but not the multiplicity) results for the case where there is just one constraint.

The vast literature on control design includes many stabilization problems that are relevant to our work. A simple one is given by [1, Example 7.4.4], which considers a family of monic cubic polynomials whose coefficients depend affinely on just one parameter, or equivalently, are determined by two affine constraints, with the requirement that all the roots lie in the unit disk (Schur stability, in the language of control). For an application in signal processing, see [13], where the authors consider the design of an infinite impulse response filter, a rational function $b(z) / a(z)$, where $a$ and $b$ are polynomials. This paper compares several different methods for optimizing the coefficients of $a$ and $b$, when their degrees are fixed, subject to constraints, including the constraint that $a$ must be Schur stable. Another applied example is a robot stabilization problem studied in $[24,16]$. More references to relevant literature in control are given in Section 4, where a specific stabilization example illustrating the results in our paper is studied in detail.

The root radius is one of a class of functions known as polynomial root max functions. A great deal is known about variational analysis of these functions $[9,7]$. That analysis focuses on the challenges presented by the non-Lipschitz behavior of these functions around polynomials with multiple active roots, a phenomenon studied in one variable by Newton using his "Newton diagram" or "Newton polygon" (see [20]). However, the focus there is on describing the variational properties of root max functions when the active root multiplicities are known. The present work is concerned with what active root structure is possible at minimizers.

The paper is organized as follows. We start in Section 2 by proving the simplest case of our main result, namely, when the number of affine constraints $k$ is just one less than the polynomial degree $n$ and the coefficients are restricted to be real. Section 3 then presents our main result for optimization of the root radius, applicable to both real and complex coefficients. Section 4 presents some examples that illustrate our results, including a detailed example arising in feedback control. Section 5 gives the proof of our main result, including the necessary preliminary material. Section 6 makes some concluding remarks. 


\section{The Simplest Case}

Let $\mathcal{P}_{n}^{1}(\mathbb{R})$ denote the affine space of monic degree $n$ polynomials with real coefficients, where $n \geq 2$. Let $\rho$ denote the root radius of a polynomial, that is, the largest of the moduli of its roots. We consider minimizing $\rho$ over polynomials in $\mathcal{P}_{n}^{1}(\mathbb{R})$ subject to $k$ linearly independent affine constraints on their coefficients. The simplest case is where $k=n-1$. A root $\lambda$ of $p$ is active relative to $\rho$ if $|\lambda|=\rho(p)$, otherwise it is inactive.

Proposition 1 Consider the problem

$$
\inf _{p \in \mathcal{P}_{n}^{1}(\mathbb{R})} \rho(p),
$$

subject to $n-1$ linearly independent affine constraints on the coefficients. Then there exists an optimal solution $p_{*}$ that has at least two active roots.

Proof Imposing $n-1$ linearly independent affine constraints on a monic real polynomial of degree $n \geq 2$ is equivalent to parametrizing the coefficients with just one variable, that is, the polynomial $p$ is in the set

$$
\left\{p(z, \lambda)=p_{0}+\lambda p_{1}: \lambda \in \mathbb{R}\right\}
$$

where $p_{0}$ is a fixed monic polynomial of degree $n$ and $p_{1}$ is a fixed nonzero polynomial with degree at most $n-1$, not necessarily monic. For each $\lambda$, let $\gamma_{1}(\lambda), \ldots, \gamma_{n}(\lambda)$ denote the roots of $p(z, \lambda)$, repeated according to multiplicity, and ordered first by highest-to-lowest modulus and second by lowest-to-highest angle, where angles are measured from the positive real axis. Thus, we have the factorization

$$
p(z, \lambda)=p_{0}(z)+\lambda p_{1}(z)=\prod_{i=1}^{n}\left(z-\gamma_{i}(\lambda)\right)
$$

and the minimization problem becomes

$$
\inf _{\lambda \in \mathbb{R}} \rho(p(z, \lambda)) \equiv \inf _{\lambda \in \mathbb{R}}\left|\gamma_{1}(\lambda)\right| .
$$

We claim that there exists an optimal polynomial with at least $n-k+1=2$ active roots, that is, at least two roots attaining the maximum modulus value. Since the lower level sets of $\rho$ are compact, an optimal polynomial $p_{*}=p\left(z, \lambda^{*}\right)$ exists, and by definition, $p_{*}$ has at least one active root, $\gamma_{1}\left(\lambda^{*}\right)$. If $\left|\gamma_{1}\left(\lambda^{*}\right)\right|=0$, then all of the roots of $p$ are zero and there is nothing more to show. So suppose $\left|\gamma_{1}\left(\lambda^{*}\right)\right|>0$. If the multiplicity of $\gamma_{1}\left(\lambda^{*}\right)$ is at least two, then there are at least two active roots, and if $\gamma_{1}\left(\lambda^{*}\right)$ is complex, then its complex conjugate must also be a root of $p(z, \lambda)$, which implies there are at least two active roots. In either case there is nothing more to show. So we may assume that $\gamma_{1}\left(\lambda^{*}\right)$ is a simple real root, which is therefore differentiable with respect to $\lambda$ at $\lambda^{*}$. We 
have $\gamma_{1}\left(\lambda^{*}\right)=\left|\gamma_{1}\left(\lambda^{*}\right)\right|$ or $\gamma_{1}\left(\lambda^{*}\right)=-\left|\gamma_{1}\left(\lambda^{*}\right)\right|$, so that the first-order optimality condition $\partial_{\lambda \mid \lambda=\lambda^{*}}\left(\left|\gamma_{1}(\lambda)\right|\right)=0$ implies that

$$
\partial_{\lambda \mid \lambda=\lambda^{*}}\left(\gamma_{1}(\lambda)\right)=0
$$

Differentiating (1) with respect to $\lambda$ on both sides, evaluating the result at $\lambda=\lambda^{*}$, and applying (2) leads to

$$
p_{1}(z)=\left(z-\gamma_{1}\left(\lambda^{*}\right)\right) r_{1}(z)
$$

where

$$
r_{1}(z):=\partial_{\lambda \mid \lambda=\lambda^{*}} \prod_{i=2}^{n}\left(z-\gamma_{i}(\lambda)\right) .
$$

Therefore,

$$
p\left(z, \lambda^{*}\right)=p_{0}(z)+\lambda^{*} p_{1}(z)=p_{0}(z)+\lambda^{*}\left(z-\gamma_{1}\left(\lambda^{*}\right)\right) r_{1}(z) .
$$

Substituting $z=\gamma_{1}\left(\lambda^{*}\right)$ yields $p_{0}\left(\gamma_{1}\left(\lambda^{*}\right)\right)=0$, which implies that $p_{0}(z)$ also has a root at $\gamma_{1}\left(\lambda^{*}\right)$. Therefore,

$$
p(z, \lambda)=\left(z-\gamma_{1}\left(\lambda^{*}\right)\right)\left(r_{0}(z)+\lambda r_{1}(z)\right)
$$

where $r_{0}(z)$ is such that $p_{0}(z)=\left(z-\gamma_{1}\left(\lambda^{*}\right)\right) r_{0}(z)$. Note that $\rho\left(r_{0}(z)+\right.$ $\left.\lambda^{*} r_{1}(z)\right)<\left|\gamma_{1}\left(\lambda^{*}\right)\right|$, since otherwise $p_{*}$ would already have two active roots. We also have

$$
\liminf _{|\lambda| \rightarrow \infty} \rho\left(r_{0}(z)+\lambda r_{1}(z)\right)=\infty
$$

since the lower level sets of $\rho$ are compact and $r_{1}$ cannot be zero as $p_{1}$ is not zero. Therefore, by continuity there must exist some $\tilde{\lambda} \in \mathbb{R}$ for which

$$
\rho\left(r_{0}(z)+\tilde{\lambda} r_{1}(z)\right)=\left|\gamma_{1}\left(\lambda^{*}\right)\right|
$$

Then the polynomial

$$
\left(z-\gamma_{1}\left(\lambda^{*}\right)\right)\left(r_{0}(z)+\tilde{\lambda} r_{1}(z)\right)=p_{0}(z)+\tilde{\lambda} p_{1}(z)
$$

attains the optimal radius $\left|\gamma_{1}\left(\lambda^{*}\right)\right|=\rho\left(p_{*}\right)$ and has at least two active roots.

Remark 1 This result can be similarly derived for polynomials with complex coefficients, but we omit the details for brevity and move on to the general case. 


\section{Main Result}

Let $n \in \mathbb{N}$ be fixed. Let $\mathcal{P}_{n}(\mathbb{F})$ denote the vector space of polynomials of degree $n$ or less over $\mathbb{F}$, and let $\mathcal{P}_{n}^{1}(\mathbb{F}) \subset \mathcal{P}_{n}(\mathbb{F})$ denote the affine subspace of monic polynomials of degree $n$ over $\mathbb{F}$, where $\mathbb{F}$ is the field $\mathbb{C}$ or $\mathbb{R}$. The polynomial root radius $\rho$ is a mapping from $\mathcal{P}_{n}^{1}(\mathbb{F})$ to $\mathbb{R}$ defined by

$$
\rho(p)=\max \{|\zeta|: p(\zeta)=0\} .
$$

It is non-convex and non-locally-Lipschitz, as may be seen by consideration of the polynomial family $\left\{p_{\varepsilon}(z): p_{\varepsilon}(z)=z^{n}-\varepsilon, \varepsilon \geq 0\right\}$. Then $\rho\left(p_{\varepsilon}\right)=\sqrt[n]{\varepsilon}$, so the root radius is not convex and is not locally Lipschitz at 0 for $n>1$. We say a root $\zeta$ of $p$ is active relative to $\rho$ if

$$
|\zeta|=\rho(p) .
$$

Otherwise $\zeta$ is inactive relative to $\rho$, which corresponds to the case where $|\zeta|<\rho(p)$.

Consider the problem

$$
\inf _{p \in \mathcal{A}_{\mathbb{F}}} \rho(p),
$$

where $\mathcal{A}_{\mathbb{F}} \subset \mathcal{P}_{n}^{1}(\mathbb{F})$ is an affine subset of dimension $n-k$, defined by $k$ linearly independent affine constraints on the coefficients of $p$.

Theorem 1 (Optimal root radius over real and complex coefficients subject to affine constraints) Let $n \in \mathbb{N}$ and $k \in\{1, \ldots, n\}$. Consider the root radius minimization problem (3) with the feasible set $\mathcal{A}_{\mathbb{F}}$, where $\mathbb{F}$ is $\mathbb{R}$ or $\mathbb{C}$ and $k$ is the number of linearly independent affine constraints, so that $\operatorname{dim}\left(\mathcal{A}_{\mathbb{F}}\right)=n-k$. Then there exists an optimal solution with at least $n-k+1$ active roots, or equivalently, with at most $k-1$ inactive roots.

We defer the proof of Theorem 1, which is fairly long and technical, to Section 5. Here we briefly mention some geometric properties that will be useful later. Define the closed $\sigma$-Schur stable set in $\mathcal{P}_{n}^{1}(\mathbb{F})$ by

$$
S_{\sigma}^{\mathbb{F}, \rho}=\left\{p \in \mathcal{P}_{n}^{1}(\mathbb{F}): \rho(p) \leq \sigma\right\},
$$

and denote its boundary by

$$
\operatorname{bdry}\left(S_{\sigma}^{\mathbb{F}, \rho}\right)=\left\{p \in \mathcal{P}_{n}^{1}(\mathbb{F}): \rho(p)=\sigma\right\} .
$$

Note that $S_{\sigma}^{\mathbb{F}, \rho}$ is compact for all $\sigma \in \mathbb{R}$ and that it is nonempty if and only if $\sigma \geq 0$. It is known that the boundary of the Schur stable set consists of ruled surfaces [14], [17, Section 4.1.3], as illustrated by Figure 1 for the case $\mathbb{F}=\mathbb{R}$, $n=3$ and $\sigma=1 / 2$. (A similar illustration appears in [17] for $\sigma=1$.)

For $\sigma=0$ the closed $\sigma$-Schur stable set is a singleton, consisting only of the polynomial $z^{n}$. Clearly, $S_{\sigma_{1}}^{\mathbb{F}, \rho} \subseteq S_{\sigma_{2}}^{\mathbb{F}, \rho}$ for all $\sigma_{1} \leq \sigma_{2}$. The optimization problem we consider requires finding a polynomial with a minimal root radius in the feasible set defined by the affine constraints, which is equivalent to finding the 


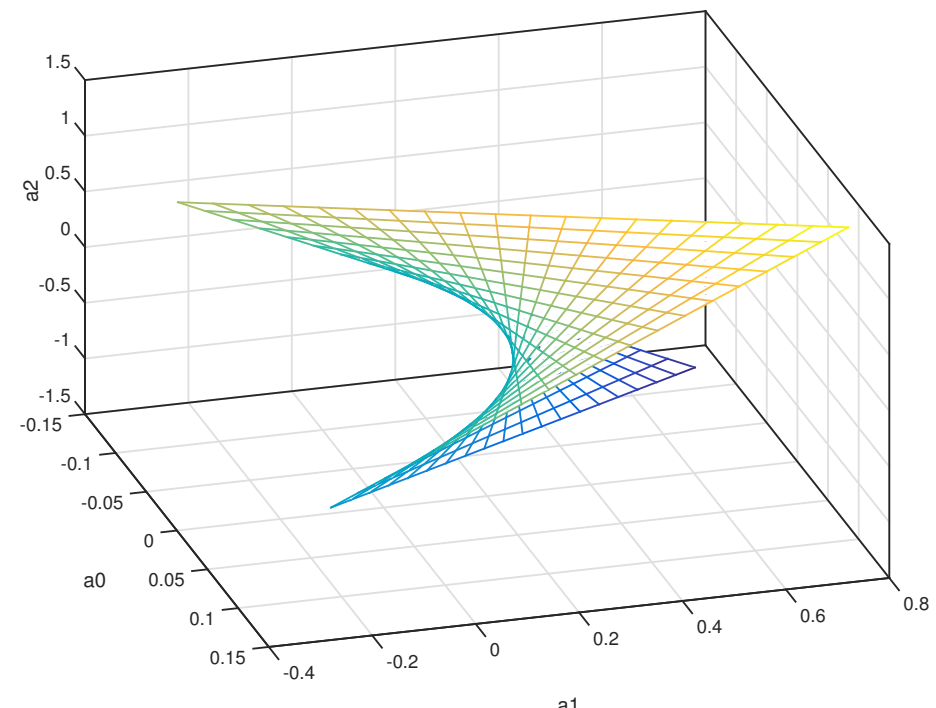

Fig. 1 An illustration of the boundary of the Schur stable set $S_{\sigma}^{\mathbb{R}, \rho}$ for $n=3$ and $\sigma=1 / 2$, identifying $\mathcal{P}_{3}^{1}(\mathbb{R})$ with $\mathbb{R}^{3}$.

smallest $\sigma$ such that the intersection of the $\sigma$-Schur stable set $S_{\sigma}^{\mathbb{F}, \rho}$ with the affine subspace $\mathcal{A}_{\mathbb{F}}$ is nonempty. Because the boundary of the $\sigma$-Schur stable set is ruled, it consists of affine sets: this is the key to the proof of Theorem 1. Given a polynomial $p \in S_{\sigma}^{\mathbb{F}, \rho} \cap \mathcal{A}_{\mathbb{F}}$ that optimizes the radius but has fewer than $n-k+1$ roots, if the dimension of an affine set containing $p$ and coinciding locally with the boundary of $S_{\sigma}^{\mathbb{F}, \rho}$ is sufficiently large, this affine set must have a nontrivial intersection with $\mathcal{A}_{\mathbb{F}}$, allowing $p$ to be perturbed in such a way that it remains feasible but the number of active roots is incremented.

\section{Examples}

4.1 Example illustrating the $n=3$ case.

This example extends [3, Example 4], for a degree 2 affine family, to $n=3$. Consider the one-dimensional affine subset

$$
\mathcal{A}_{\mathbb{R}}=\left\{z^{3}+\lambda z^{2}-\lambda z-1 \in \mathcal{P}_{1}^{3}(\mathbb{R}): \lambda \in \mathbb{R}\right\},
$$


implicitly defined by $k=2$ independent affine constraints. We find that, as a function of $\lambda$,

$$
\rho(p(z, \lambda))= \begin{cases}(-(\lambda+1)+\sqrt{(\lambda+3)(\lambda-1)}) / 2 & \text { if } \lambda<-3, \\ 1 & \text { if }-3 \leq \lambda \leq 1, \\ ((\lambda+1)+\sqrt{(\lambda+3)(\lambda-1)}) / 2 & \text { if } 1<\lambda,\end{cases}
$$

which has a global minimum value of 1 for all $\lambda \in[-3,1]$. This example is consistent with the theory since an optimizer exists with at least two active roots (in fact, two such optimizers exist, namely -3 and 1 ). The graph of the radius versus the parameter $\lambda$ is shown in Figure 2 .

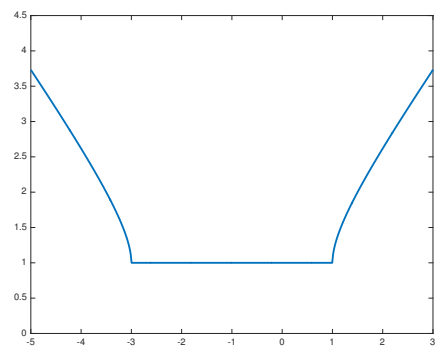

Fig. 2 The polynomial root radius of $\left\{z^{3}+\lambda z^{2}-\lambda z-1 \in \mathcal{P}_{1}^{3}(\mathbb{R}): \lambda \in \mathbb{R}\right\}$.

\subsection{Examples from Feedback Control}

Let $a \in \mathcal{P}_{d_{a}}^{1}(\mathbb{R})$ and $b \in \mathcal{P}_{d_{b}}(\mathbb{R})$ be given, with $d_{a}>d_{b}$. In control one refers to the rational function $b / a$ as a plant or open loop system that is strictly proper as $d_{a}>d_{b}$. In feedback control design, one wishes to construct another rational function $y / x$, called a controller, so that the closed loop system, modeled by the rational function $q / p \equiv b x /(a x+b y)[6,17,23]$ has desired properties, most notably with poles, that is roots of $p=a x+b y$, in the relevant stability region. Let $x \in \mathcal{P}_{d_{x}}^{1}(\mathbb{R})$ and $y \in \mathcal{P}_{d_{y}}(\mathbb{R})$ with $y / x$ proper, that is $d_{x} \geq d_{y}$, and write

$$
\begin{aligned}
& a(z)=z^{d_{a}}+a_{d_{a}-1} z^{d_{a}-1}+\cdots+a_{0}, \quad b(z)=b_{d_{b}} z^{d_{b}}+b_{d_{b}-1} z^{d_{b}-1}+\cdots+b_{0}, \\
& x(z)=z^{d_{x}}+x_{d_{x}-1} z^{d_{x}-1}+\cdots+x_{0}, \quad y(z)=y_{d_{y}} z^{d_{y}}+y_{d_{y}-1} z^{d_{y}-1}+\cdots+y_{0} .
\end{aligned}
$$

Then $a x$ is the monic polynomial

$z^{d_{p}}+\left(a_{d_{a}-1}+x_{d_{x}-1}\right) z^{d_{p}-1}+\left(a_{d_{a}-2}+a_{d_{a}-1} x_{d_{x}-1}+x_{d_{x}-2}\right) z^{d_{p}-2}+\cdots+a_{0} x_{0}$ with degree $d_{p}=d_{a}+d_{x}$, and with coefficients depending affinely on the $d_{x}$ variables $x_{d_{x}-1}, \ldots, x_{0}$. Clearly, the polynomial $p=a x+b y$ also has degree 


\begin{tabular}{|c|c|c|c|c|c|}
\hline$d$ & $\begin{array}{c}n \\
(d+5)\end{array}$ & $\begin{array}{c}\text { \#vars } \\
(2 d+1)\end{array}$ & $\begin{array}{c}\text { \#constr } \\
(4-d)\end{array}$ & $\begin{array}{c}\min \text { active } \\
(\min (2 d+2, n))\end{array}$ & actual active \\
\hline 0 & 5 & 1 & 4 & 2 & 3 \\
1 & 6 & 3 & 3 & 4 & 6 \\
2 & 7 & 5 & 2 & 6 & 6 \\
3 & 8 & 7 & 1 & 8 & 8 \\
4 & 9 & 9 & 0 & 9 & 9 \\
\hline
\end{tabular}

Table 1 Experiment with $a(z)=z^{5}+z^{3}-z+2$ and $b(z)=z^{2}+1$

$d_{p}$ and depends affinely on the $d_{x}+d_{y}+1$ variables $x_{d_{x}-1}, \ldots, x_{0}, y_{d_{y}}, \ldots, y_{0}$. Furthermore, it is not hard to show that as long as $a$ and $b$ have no nonconstant common factors, then the mapping $(x, y) \mapsto a x+b y$ is one-to-one. It follows that if we now minimize the root radius of $p$ over these variables, we have an optimization problem of the form $(3)$, with $n=d_{p}$ and

$$
k=\left(d_{a}+d_{x}\right)-\left(d_{x}+d_{y}+1\right)=d_{a}-d_{y}-1
$$

affine constraints on the coefficients of $p$.

We do not have a method to find global minimizers of root max function optimization problems so we approximated them using a local optimization method, as was done in [22] for matrix eigenvalue optimization problems. As explained in [18], the quasi-Newton method known as BFGS, which originated in 1970 to minimize differentiable functions [21], is also extremely effective for finding local minimizers of nonsmooth functions, particularly in the locally Lipschitz case, but also including non-Lipschitz functions such as the root radius, although the same accuracy cannot be expected in the latter case. So, to search for minimizers of $\rho(p)$, where $p$ is given above, we ran BFGS from 1000 randomly generated starting points for each problem instance, with the hope that for small problem instances, global minima will be found, although as noted below in Remark 3, our result on root activity also applies to local minima. The experiments were conducted using MATLAB [19].

Let $a(z)=z^{5}+z^{3}-z+2$ and let $b(z)=z^{2}+1$, and let us restrict attention to the case $d_{x}=d_{y}$, which we denote by $d$ for brevity. We consider problem instances with $d$ ranging from 0 to 4 . Table 1 shows the corresponding values for $n=d_{p}=d_{a}+d=d+5$, the number of variables $2 d+1$, the number of constraints $k=(d+5)-(2 d+1)=4-d$, the minimum number of active roots of the optimal polynomial ${ }^{1}$ according to Theorem 1 , namely $n-k+1=2 d+2$, and the actual number of active roots (within a tolerance) of the polynomial with least root radius found by BFGS. We see that in each case, the computational results are consistent with the theory.

For each value of $d$, Figure 4.2 shows the actual roots of the computed optimal polynomial $\tilde{p}$ together with the associated circle of radius $\rho(\tilde{p})$. We see that for $d=0$, there are two inactive roots, and for $d=2$, there is one

\footnotetext{
1 Assuming that it is unique. The instance with $d=4$ reported in the last row of the table has no constraints, so the optimal root radius is zero; hence in this case the minimal number of active roots is $n=9$.
} 


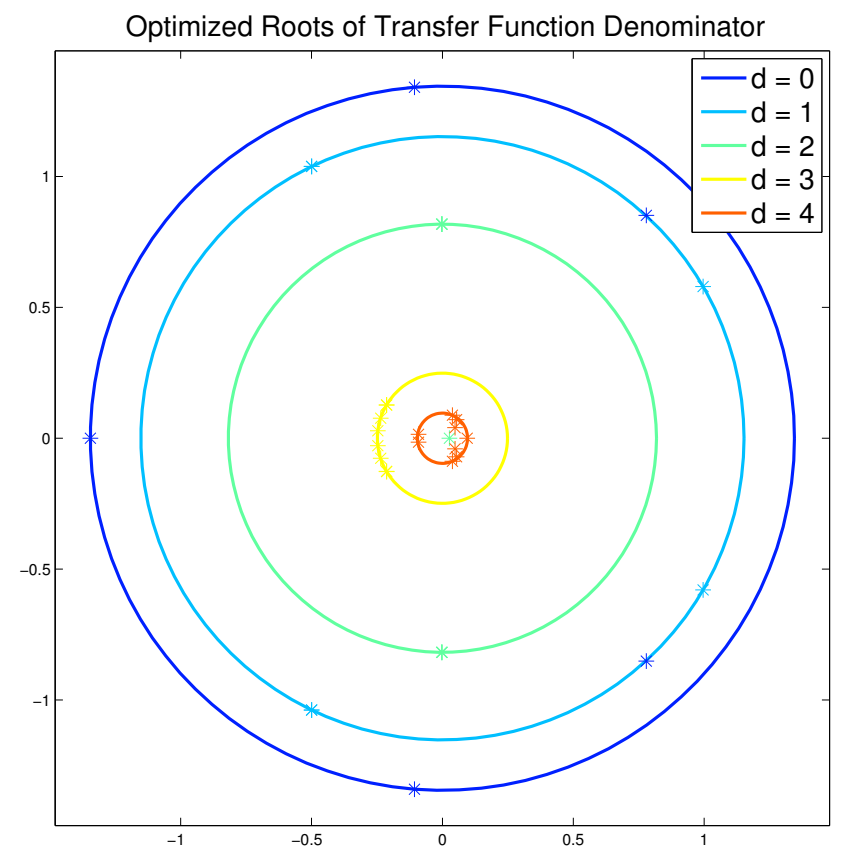

Fig. 3 Optimized Roots of Transfer Function Denominator

inactive root; otherwise all roots are active within a small tolerance. Note that for each $d>0$, some of the optimal roots are coincident, but the number varies, and when $d=0$, all five optimal roots are distinct. In the case $d=3$, with just one constraint, the optimal root radius found by BFGS agrees well with the known globally optimal value obtained using the algorithm described in [3]. In this case, all the computed roots are clustered near one real number, although one cannot expect them to be exactly coincident, as they are in theory, given the known sensitivity of multiple roots (in this case with multiplicity 8) to small numerical errors. In the case $d=4$, when there are no constraints, the optimal polynomial is $z^{9}$ with all roots at zero, but again, but we cannot expect to be able to compute this very accurately.

Finally, note from Figure 4.2 that when $d$ is increased, the optimal root radius decreases. This must always be the case, as a simple argument shows. Suppose $x, y$ are optimal for a given degree $d$. If we now increase $d$ by one, then the polynomial $z(a(z) x(z)+b(z) y(z))$ has the same roots as the previous optimal polynomial $a(z) x(z)+b(z) y(z)$ along with one additional root at zero. So, it has the same radius, and since $z(x(z))$ is monic, optimizing over $x(z)$ and $y(z)$ with increased degree can only reduce the radius further, not increase it. 


\section{Proofs}

This section has four parts. In the first three subsections, we work with polynomial root max functions in general, not only the root radius, partly for increased generality, but also to facilitate possible future work extending our results from the root radius to other polynomial root max functions such as the abscissa (see below). In Section 5.1 we define these functions and explain the feasible set representations that we will be using in the proofs. In Section 5.2 we establish a preliminary lemma in linear algebra that will be useful. In Section 5.3 we provide a key lemma on the effect of perturbing the coefficients of an inactive factor of a polynomial, where the activity is relative to the particular polynomial root max function under consideration. In Section 5.4 we provide the proof of Theorem 1.

\subsection{Definitions}

\subsubsection{Polynomial root max functions}

Let $n \in \mathbb{N}$ be fixed. As earlier, let $\mathcal{P}_{n}(\mathbb{F})$ denote the vector space of polynomials of degree $n$ or less over $\mathbb{F}$, and let $\mathcal{P}_{n}^{1}(\mathbb{F}) \subset \mathcal{P}_{n}(\mathbb{F})$ denote the affine subspace of monic polynomials of degree $n$ over $\mathbb{F}$, where $\mathbb{F}$ is the field $\mathbb{C}$ or $\mathbb{R}$.

Let $f: \mathbb{C} \rightarrow \mathbb{R}$. The function $f$ induces a function $\varphi_{f}: \mathcal{P}_{n}(\mathbb{F}) \rightarrow \mathbb{R}$ in the following way:

$$
\varphi_{f}(p)=\max \{f(\zeta): p(\zeta)=0\}
$$

We call $\varphi_{f}$ a polynomial root max function, or simply root max function. If $f$ is continuous, so is $\varphi_{f}$. Two polynomial root max functions that frequently arise in applications are the polynomial root radius $\rho: \mathcal{P}_{n}^{1}(\mathbb{F}) \rightarrow \mathbb{R}$, given by

$$
\rho(p)=\max \{|\zeta|: p(\zeta)=0\},
$$

and the polynomial root abscissa $\alpha: \mathcal{P}_{n}^{1}(\mathbb{F}) \rightarrow \mathbb{R}$, given by

$$
\alpha(p)=\max \{\operatorname{Re}(\zeta): p(\zeta)=0\} .
$$

Polynomial root max functions are, in general, non-convex and non-locallyLipschitz, as noted in Section 3. We say a root $\zeta$ of $p$ is active relative to $\varphi_{f}$ if

$$
f(\zeta)=\varphi_{f}(p) .
$$

Otherwise $\zeta$ is inactive relative to $\varphi_{f}$, which corresponds to the case where $f(\zeta)<\varphi_{f}(p)$. 


\subsubsection{Feasible set representations}

Let $k \in\{1, \ldots, n\}$ be a fixed number of affine constraints on the coefficients of monic polynomials of degree $n$. We represent the affine constraints as follows. Let

$$
\mathcal{A}_{\mathbb{F}}=\left\{\begin{array}{ll}
z^{n}+a_{n-1} z^{n-1}+\cdots+a_{1} z+a_{0}: & B_{n}^{(\ell)}+\sum_{i=0}^{n-1} B_{i}^{(\ell)} a_{i}=0 \\
& \text { for } \ell=1, \ldots, k \\
& \text { where } a_{i} \in \mathbb{F} \\
\text { for } i=0, \ldots, n-1 .
\end{array}\right\}
$$

denote the affine subspace of $\mathcal{P}_{n}^{1}(\mathbb{F})$ satisfying the $k$ constraints. We assume the $k$ affine constraints are linearly independent so that the dimension of $\mathcal{A}_{\mathbb{F}}$ is $n-k$.

An alternative representation of $\mathcal{A}_{\mathbb{F}}$ is in the coefficient space $\mathbb{F}^{n}$ as follows. Let $p(z)=z^{n}+a_{n-1} z^{n-1}+\cdots+a_{1} z+a_{0}$. Then $p \in \mathcal{A}_{\mathbb{F}}$ if and only if $B a=b$, where $a=\left(a_{0}, \ldots, a_{n-1}\right)^{T}$,

$$
B=\left[\begin{array}{cccc}
B_{0}^{(1)} & B_{1}^{(1)} & \cdots & B_{n-1}^{(1)} \\
B_{0}^{(2)} & B_{1}^{(2)} & \cdots & B_{n-1}^{(2)} \\
\vdots & \vdots & \ddots & \vdots \\
B_{0}^{(k)} & B_{1}^{(k)} & \cdots & B_{n-1}^{(k)}
\end{array}\right], \quad \text { and } \quad b=\left[\begin{array}{c}
-B_{n}^{(1)} \\
-B_{n}^{(2)} \\
\vdots \\
-B_{n}^{(k)}
\end{array}\right] .
$$

The requirement that $\operatorname{dim}\left(\mathcal{A}_{\mathbb{F}}\right)=n-k$ is equivalent to the condition $\operatorname{rank}(B)=$ $k$. Let $\mathbb{A} \subset \mathbb{F}^{n}$ denote the set of feasible coefficients:

$$
\mathbb{A}=\{a: B a-b=0\} .
$$

We use both representations of the feasible set.

Remark 2 It is useful to keep in mind that in either representation (6) or (7), the entries $B_{i}^{(\ell)}$ are fixed and the coefficients $a_{0}, \ldots, a_{n-1}$ are free. The subscript in $\mathcal{A}_{\mathbb{F}}$ identifies the relevant field.

We are interested in problems of the form

$$
\inf _{p \in \mathcal{A}_{\mathbb{F}}} \varphi_{f}(p),
$$

where $\mathbb{F}$ is $\mathbb{R}$ or $\mathbb{C}$. Our work is concerned with the number of active and inactive roots of optimal solutions to this problem.

\subsection{Preliminary results for linear transformations}

Lemma 1 (Linear transformation rank lemma) Let $k, n \in \mathbb{N}$ and $r \in$ $\mathbb{N} \cup\{0\}$ with $r \leq k<n$. Let $F: \mathbb{F}^{k} \rightarrow \mathbb{F}^{n}$ and $G: \mathbb{F}^{n} \rightarrow \mathbb{F}^{k}$ be linear transformations with $\operatorname{rank}(F)=\operatorname{rank}(G)=k$. Then $\operatorname{dim}(\operatorname{range}(F) \cap \operatorname{null}(G))=r$ if and only if $\operatorname{rank}(G \circ F)=k-r$. 
Proof First note that $\operatorname{rank}(G \circ F)=k-r$ is equivalent to $\operatorname{dim}(\operatorname{null}(G \circ F))=r$. Next since $\operatorname{rank}(F)=k, F$ is injective. Define $\widetilde{F}: \mathbb{F}^{k} \rightarrow \operatorname{range}(F)$, that is, the map $F$ but with image space given by the range $(F)$ instead of $\mathbb{F}^{n}$. Then $\widetilde{F}$ is an isomorphism. Define $S_{1}=\operatorname{null}(G \circ F)$ and $S_{2}=\widetilde{F}^{-1}(\operatorname{range}(F) \cap \operatorname{null}(G))$. We claim that these sets are equal. First suppose $w \in S_{2}$. Then $w=\widetilde{F}^{-1}(v)$ for some $v$ with $G(v)=0$. Moreover, $v=\widetilde{F}(w)=F(w)$ and thus $(G \circ F)(w)=0$, which implies that $w \in S_{1}$. Therefore $S_{2} \subset S_{1}$. On the other hand, suppose $w \in S_{1}$. It follows that $(G \circ F)(w)=0$. Let $v=F(w)$. Then $v \in \operatorname{null}(G)$ and $v \in \operatorname{range}(F)$, which implies $\widetilde{F}^{-1}(v)=w \in S_{2}$. Therefore $S_{1} \subset S_{2}$. So $S_{1}=S_{2}$, establishing the desired result.

Corollary 1 Let $k, n \in \mathbb{N}$ and $r \in \mathbb{N} \cup\{0\}$ with $r \leq k<n$. Let $F: \mathbb{F}^{k} \rightarrow \mathbb{F}^{n}$ and $G: \mathbb{F}^{n} \rightarrow \mathbb{F}^{k}$ be linear transformations with $\operatorname{rank}(F)=\operatorname{rank}(G)=k$. Let $A_{F} \subset \mathbb{F}^{n}$ be an affine subspace that is a translate of range $(F)$ by a fixed vector and let $A_{G} \subset \mathbb{F}^{n}$ be an affine subspace that is a translate of $\operatorname{null}(G)$ by the same vector. Then

$$
\operatorname{dim}\left(A_{F} \cap A_{G}\right)=r
$$

if and only if

$$
\operatorname{rank}(G \circ F)=k-r
$$

where the dimension of an affine subspace $A \subset \mathbb{F}^{n}$ is taken to be $\operatorname{dim}(A-\{a\})$ for any $a \in A$.

5.3 Results for perturbing the coefficients of a polynomial factor with inactive roots

Let $f: \mathbb{C} \rightarrow \mathbb{R}$ be a continuous function, and let $\varphi_{f}: \mathcal{P}_{n}^{1}(\mathbb{F}) \rightarrow \mathbb{R}$ be its associated root max function, as defined in (4). Let $\mathcal{A}_{\mathbb{F}} \subset \mathcal{P}_{n}^{1}(\mathbb{F})$ be as defined in (5), so that $\operatorname{dim}\left(\mathcal{A}_{\mathbb{F}}\right)=n-k$, where the number of constraints $k$ satisfies $1 \leq k<n$. Note that the case $k=n$ is trivial since the feasible set reduces to a single point.

Let $p_{*} \in \mathcal{A}_{\mathbb{F}}$ where

$$
p_{*}(z)=z^{n}+a_{n-1}^{*} z^{n-1}+\cdots+a_{0}^{*}
$$

with function value

$$
\gamma_{*}:=\varphi_{f}\left(p_{*}\right) .
$$

Since $p_{*} \in \mathcal{A}_{\mathbb{F}}$, we have $B a^{*}=b$, where $B$ and $b$ are defined in (6). Suppose further that $p_{*}$ has

$$
n_{I} \geq k
$$

inactive roots and

$$
n_{A}:=n-n_{I}
$$

active roots. Factor $p_{*}$ according to root activity as

$$
p_{*}(z)=q^{A}(z) q^{I}(z)
$$


where $q^{A}(z)$ is a polynomial of degree $n_{A}$ whose roots are all active, and $q^{I}(z)$ is a polynomial of degree $n_{I} \geq k$ whose roots are all inactive. Express $q^{I}(z)$ by

$$
q^{I}(z)=z^{n_{I}}+q_{n_{I}-1} z^{n_{I}-1}+\cdots+q_{0} .
$$

Let $\Delta \in \mathbb{F}^{k}$, with components indexed from 0 to $k-1$, and make linear perturbations to the $k$ lowest order coefficients of $q^{I}$ as follows:

$$
q_{\Delta}^{I}(z)=z^{n_{I}}+q_{n_{I}-1} z^{n_{I}-1}+\cdots+q_{k} z^{k}+\left(q_{k-1}+\Delta_{k-1}\right) z^{k-1}+\cdots+\left(q_{0}+\Delta_{0}\right),
$$

if $n_{I}>k$, and

$$
q_{\Delta}^{I}(z)=z^{k}+\left(q_{k-1}+\Delta_{k-1}\right) z^{k-1}+\cdots+\left(q_{0}+\Delta_{0}\right)
$$

if $n_{I}=k$. Multiplying $q_{\Delta}^{I}(z)$ by $q^{A}(z)$ yields linear perturbations of the coefficients of $p_{*}(z)$ :

$$
p_{\Delta}(z)=q^{A}(z) q_{\Delta}^{I}(z)=z^{n}+a_{n-1}(\Delta) z^{n-1}+\cdots+a_{0}(\Delta) .
$$

We can therefore express the inactive-root coefficient perturbation map $C^{I}$ : $\mathbb{F}^{k} \rightarrow \mathbb{F}^{n}$ by

$$
C^{I}(\Delta)=a^{*}+M \Delta
$$

so that each coefficient is perturbed as

$$
a_{i}(\Delta)=a_{i}^{*}+(M \Delta)_{i}
$$

for $i=0, \ldots, n-1$, where $M \in \mathbb{F}^{n \times k}$ (whose rows and columns are indexed from 0 to $n-1$ and 0 to $k-1$, respectively), and $C^{I}(0)=\left(a_{0}^{*}, a_{1}^{*}, \ldots, a_{n-1}^{*}\right)^{T}$ are the coefficients of $p_{*}(z)$ as in $(8)$. Since $f$ is continuous, small perturbations of $q^{I}$ do not affect the active part, that is,

$$
\varphi_{f}\left(p_{*}\right)=\varphi_{f}\left(p_{\Delta}\right)
$$

for small $\Delta$. Let $H \subset \mathbb{F}^{n}$ denote the image of $C^{I}$, that is,

$$
H:=\left\{a^{*}+M v: v \in \mathbb{F}^{k}\right\}
$$

which is an affine subspace of $\mathbb{F}^{n}$ of dimension less than or equal $k$.

Lemma 2 (Inactive perturbation, I) Let $f$ and $\varphi_{f}$ be as in (4), with $f$ continuous. Let $p_{*} \in \mathcal{A}_{\mathbb{F}}$, and let $\gamma_{*}, q^{I}, q_{\Delta}^{I}, C^{I}$, and $H$ be as in (8)-(17). Then

$$
\operatorname{rank}(M)=k,
$$

so that

$$
\operatorname{dim}(H)=k .
$$


Proof Note that we consider dimension over $\mathbb{F}$. Let $\Delta \in \mathbb{F}^{k}$ be fixed. Straightforward computations show that for $M \in \mathbb{F}^{n \times k}$, given in (16),

$$
\begin{aligned}
M \Delta=0 & \Longleftrightarrow M \Delta+a^{*}=a^{*} \\
& \Longleftrightarrow p_{\Delta}=p_{*} \\
& \Longleftrightarrow q_{\Delta}^{I}(z)-q^{I}(z)=0 \quad \forall z \\
& \Longleftrightarrow \Delta_{k-1} z^{k-1}+\cdots+\Delta_{0}=0 \quad \forall z \\
& \Longleftrightarrow \Delta=0,
\end{aligned}
$$

which implies that $\operatorname{dim} \operatorname{null}(M)=0$, that is, $\operatorname{rank}(M)=k$, which establishes (18).

Corollary 2 (Inactive perturbation, II) Let $f$ and $\varphi_{f}$ be as in (4), with $f: \mathbb{C} \rightarrow \mathbb{R}$ continuous. Let $p_{*} \in \mathcal{A}_{\mathbb{F}}$, and let $\gamma_{*}, q^{I}, q_{\Delta}^{I}, C^{I}$, and $H$ be as in (8)-(17). Then

$$
\operatorname{dim}(\mathbb{A} \cap H)=r \Longleftrightarrow \operatorname{rank}(B M)=k-r
$$

where $B$ and $\mathbb{A}$ are given in (6) and (7), and where $\operatorname{dim}(\mathbb{A} \cap H)$ means the dimension of the subset $\mathbb{A} \cap H-\left\{a^{*}\right\}$ where $a^{*}=\left(a_{0}^{*}, a_{1}^{*}, \ldots, a_{n-1}^{*}\right)^{T}$ is given in (8) and $0 \leq r \leq k$.

Proof This follows from Corollary 1 and Lemma 2.

Our primary interest in Corollary 2 is in the cases $r=0$ and $r=k$, which will be used to prove the results in the following section.

\subsection{Proof of Theorem 1}

The idea behind the proof of Theorem 1 is as follows. Given an optimal polynomial, with radius $\gamma_{*}$ and fewer than $n-k+1$ active roots, we factor it according to root activity. We then perturb the coefficients of the inactive factor until one of its roots becomes active. More specifically, we show the existence of a line contained in the feasible set $\mathcal{A}_{\mathbb{F}}$ along which the optimal polynomial can be perturbed, remaining on the boundary of the $\gamma_{*}$-Schur stable set until the perturbation is sufficiently large that an inactive root becomes active. Then this process is repeated as needed.

Proof Since $\mathcal{A}_{\mathbb{F}}$ is nonempty, a feasible polynomial exists. By the compactness of $S_{\sigma}^{\mathbb{F}, \rho}$ for each $\sigma \in \mathbb{R}$ and the continuity of $\rho$, an optimal solution exists. Let $p_{*}=z^{n}+a_{n-1}^{*} z^{n-1}+\cdots+a_{0}^{*}$ be an optimizer with optimal value $\gamma_{*}$. Since $p_{*} \in \mathcal{A}_{\mathbb{F}}$, we have $B a^{*}=b$. Note that $p_{*}$ has at least one active root and at most $n-1$ inactive roots. In the case where $k=n$, we have that $\mathcal{A}_{\mathbb{F}}=\left\{p_{*}\right\}$ and there is nothing more to show. So suppose $k<n$.

If $\rho\left(p_{*}\right)=0$, then $p_{*}=z^{n}$, which implies that every root is active, establishing the result in this case. Suppose, therefore, that $\rho\left(p_{*}\right)>0$ and that there are $n_{I} \geq k$ inactive roots and $n_{A}=n-n_{I}$ active roots (as in (10)). Factor $p_{*}$ 
according to root activity as in (11): $p_{*}(z)=q^{A}(z) q^{I}(z)$, and express $q^{I}(z)$ as in $(12)$ by $q^{I}(z)=z^{n_{I}}+q_{n_{I}-1} z^{n_{I}-1}+\cdots+q_{0}$. In the real case, where $\mathbb{F}=\mathbb{R}$, express the active part in terms of its real roots and complex conjugate pairs by

$$
q^{A}(z)=\prod_{i=1}^{n_{1}}\left(z-h_{i}\right) \prod_{i=n_{1}+1}^{n_{2}}\left(z^{2}-2 d_{i} z+\gamma_{*}^{2}\right)
$$

where

$$
\begin{aligned}
h_{i} & \in \mathbb{R},\left|h_{i}\right|=\gamma_{*} \text { for } i=1, \ldots, n_{1}, \text { and } \\
d_{i} & \in \mathbb{R},\left|d_{i}\right|<\gamma_{*} \text { for } i=n_{1}+1, \ldots, n_{2}
\end{aligned}
$$

note that $n_{1}$ and $n_{2}$ satisfy $n_{A}=n_{1}+2\left(n_{2}-n_{1}\right)$. In the complex case, where $\mathbb{F}=\mathbb{C}$, express the active part in terms of its linear factors by

$$
q^{A}(z)=\prod_{i=1}^{n_{2}}\left(z-h_{i}\right)
$$

where

$$
h_{i} \in \mathbb{C},\left|h_{i}\right|=\gamma_{*} \quad \text { for } i=1, \ldots n_{2}
$$

and we define $n_{2}:=n_{A}$ for notational consistency. Consider affine perturbations $\Delta \in \mathbb{F}^{k}$ of the coefficients of $q^{I}(z)$ as defined in (13) or (14). As in (15), multiplying $q_{\Delta}^{I}(z)$ by $q^{A}(z)$ yields $p_{\Delta}(z)$ - a family of polynomials whose coefficients are affine perturbations of those of $p_{*}(z)$ that influence only the inactive roots when $\Delta$ is small. We utilize the inactive-root coefficient perturbation map $C^{I}: \mathbb{F}^{k} \rightarrow \mathbb{F}^{n}$ as given in (16), where $C^{I}(\Delta)=a^{*}+M \Delta$ and $a_{i}(\Delta)=a_{i}^{*}+(M \Delta)_{i}$, so that $C^{I}(0)=\left(a_{0}^{*}, a_{1}^{*}, \ldots, a_{n-1}^{*}\right)^{T}$ are the coefficients of $p_{*}(z)$ as defined in (8). Let $H \subset \mathbb{F}^{n}$ be the image of $C^{I}$ as defined in (17).

By Corollary 2,

$$
\operatorname{dim}(\mathbb{A} \cap H)=0 \Longleftrightarrow \operatorname{rank}(B M)=k .
$$

In the real case, where $\mathbb{F}=\mathbb{R}$, consider perturbations $\widetilde{\Delta} \in \mathbb{R}^{n_{2}}$ of the active part

$$
q_{\widetilde{\Delta}}^{A}(z):=\prod_{i=1}^{n_{1}}\left(z-h_{i}\left(1+\widetilde{\Delta}_{i}\right)\right) \prod_{i=n_{1}+1}^{n_{2}}\left(z^{2}-2 d_{i} z+\gamma_{*}^{2}\left(1+\widetilde{\Delta}_{i}\right)\right)
$$

where $h_{i}$ and $d_{i}$ are given in (19). In the complex case, where $\mathbb{F}=\mathbb{C}$, consider perturbations $\widetilde{\Delta} \in \mathbb{C}^{n_{2}}$ of the active part

$$
q_{\widetilde{\Delta}}^{A}(z):=\prod_{i=1}^{n_{2}}\left(z-h_{i}\left(1+\widetilde{\Delta}_{i}\right)\right)
$$

where $h_{i}$ is given in (20). It is worth noting that in this proof, we perturb the roots of the active part, as opposed to the coefficients of the active part, as was done in [3, Theorem 1]. 
Let

$$
p_{\widetilde{\Delta}, \Delta}(z)=q_{\widetilde{\Delta}}^{A}(z) q_{\Delta}^{I}(z)=z^{n}+a_{n-1}(\widetilde{\Delta}, \Delta) z^{n-1}+\cdots+a_{0}(\widetilde{\Delta}, \Delta),
$$

the perturbation of $p_{*}$ obtained by perturbing both $q^{I}(z)$ and the active part $q^{A}(z)$. Define the expanded perturbed coefficient map $C: \mathbb{F}^{n_{2}} \times \mathbb{F}^{k} \rightarrow \mathbb{F}^{n}$ by

$$
C(\widetilde{\Delta}, \Delta)=\left(a_{0}(\widetilde{\Delta}, \Delta), \ldots, a_{n-1}(\widetilde{\Delta}, \Delta)\right)^{T},
$$

where each perturbed coefficient $a_{i}(\widetilde{\Delta}, \Delta)$ is multilinear in the components of $\widetilde{\Delta}$ and $\Delta$. Note that $C(0, \Delta)=C^{I}(\Delta)=a^{*}+M \Delta$, where $a^{*}$ is given in (8), and $C^{I}$ and $M$ are given in (16). Also note that $C(0,0)=a^{*}$. Define the maps $L: \mathbb{F}^{n_{2}} \times \mathbb{F}^{k} \rightarrow \mathbb{F}^{k}$ and $L^{I}: \mathbb{F}^{k} \rightarrow \mathbb{F}^{k}$ by

$$
L(\widetilde{\Delta}, \Delta)=B C(\widetilde{\Delta}, \Delta)-b \quad \text { and } \quad L^{I}(\Delta)=B C^{I}(\Delta)-b .
$$

Then $L^{I}(0)=B C^{I}(0)-b=B a^{*}-b=0$. Note that

$$
D_{L^{I}}(0)=B M
$$

the real or complex ${ }^{2}$ derivative of $L^{I}$ evaluated at 0 . The proof proceeds by making a rank argument about this derivative. The component $\Delta$ does not affect the value of $\rho\left(p_{\widetilde{\Delta}, \Delta}\right)$ provided $\Delta$ and $\widetilde{\Delta}$ are small, since $\Delta$ corresponds to perturbations of the inactive part only, the roots are continuous in the coefficients and the complex modulus function is continuous. Suppose that $\operatorname{dim}(\mathbb{A} \cap H)=0$ (in which case $\mathbb{A} \cap H=\left\{a^{*}\right\}$ ). By $(21), \operatorname{rank}(B M)=k$. This implies $\operatorname{rank}\left(D_{L^{I}}(0)\right)=k$. By the implicit function theorem ${ }^{3}$ there exists $(\widetilde{\Delta}, \Delta) \in \mathbb{F}^{n_{2}} \times \mathbb{F}^{k}$ near the origin satisfying $L(\widetilde{\Delta}, \Delta)=0$, so that $p_{\widetilde{\Delta}, \Delta}$ is feasible. Furthermore, in the real case, we can choose $\widetilde{\Delta}_{i}<0$ for all $i \in$ $\left\{1,2, \ldots, n_{2}\right\}$, so that the perturbed factors $\left(z-h_{i}\left(1+\widetilde{\Delta}_{i}\right)\right)$ for $i=1, \ldots, n_{1}$, yield roots of modulus strictly less than $\gamma_{*}$, as do the roots corresponding to the perturbed conjugate pairs $\left(z^{2}-2 d_{i} z+\gamma_{*}^{2}\left(1+\widetilde{\Delta}_{i}\right)\right)$, for $i=n_{1}+1, \ldots, n_{2}$; in the complex case, we can choose $\widetilde{\Delta}_{i}$ to be real and strictly negative for $i \in$ $\left\{1,2, \ldots, n_{2}\right\}$, so that the perturbed factors $\left(z-h_{i}\left(1+\widetilde{\Delta}_{i}\right)\right)$ for $i=1, \ldots, n_{2}$, yield roots of modulus strictly less than $\gamma_{*}$. Therefore $\rho\left(p_{\widetilde{\Delta}, \Delta}\right)<\gamma_{*}$, which contradicts the optimality of $p_{*}$. Hence $\operatorname{dim}(\mathbb{A} \cap H) \geq 1$. So there exists $v \neq 0$ such that $a^{*}+M v \in \mathbb{A} \cap H$. In fact, $a^{*}+M(t v) \in \mathbb{A} \cap H$ for all $t \in \mathbb{R}$, i.e. $C^{I}(t v) \in \mathbb{A} \cap H$. Furthermore, for $|t|$ near $0, C^{I}(t v) \in \mathbb{A} \cap H \cap \operatorname{bdry}\left(S_{\gamma_{*}}^{\mathbb{F}, \rho}\right)$.

Consider the one-parameter polynomial family

$$
r_{t}(z):=q^{A}(z) q_{\Delta}^{I}(z) \quad \text { with } \Delta=t v, t \in \mathbb{R} .
$$

By construction, $r_{t}$ is feasible for all $t$ since its coefficients lie in $\mathbb{A}$. Let $f(t)=\rho\left(q_{\Delta}^{I}\right)$, the root radius of the perturbed inactive part. Then $f$ is clearly

\footnotetext{
2 The components of $L^{I}$ are affine with respect to each complex variable and therefore their derivatives satisfy the Cauchy-Riemann equations.

3 For a complex version see [15, Theorem 7.6].
} 
continuous and $f(0)<\gamma_{*}$. The lower level sets of $\rho$ are bounded, and so $f$ is unbounded as $|t| \rightarrow \infty$. By continuity of $f$, there exists $t_{*} \in \mathbb{R}$ such that $f\left(t_{*}\right)=\gamma_{*}$. This implies that the perturbed polynomial $q_{\Delta_{*}}^{I}$, where $\Delta_{*}=t_{*} v$, has at least one active root so the polynomial

$$
p_{\text {new }}(z)=q^{A}(z) q_{\Delta_{*}}^{I}(z)
$$

has at least $n_{A}+1$ active roots, i.e., at least one more active root than $p_{*}$, while having the same root radius as $p_{*}$. Let $s$ denote the increase in the number of active roots. Then $s \geq 1$, and the number of inactive roots of $p_{\text {new }}$ equals $n_{I}-s$. If $n_{I}-s \geq k$, we can apply the same procedure to the polynomial $p_{\text {new }}$ which is re-factored to reflect the change in activity as

$$
p_{\text {new }}=q_{\text {new }}^{A} q_{\text {new }}^{I}
$$

with $\operatorname{deg}\left(q_{\text {new }}^{A}\right)=n_{A}+s$ and $\operatorname{deg}\left(q_{\text {new }}^{I}\right)=n-\left(n_{A}+s\right)$. This process is repeated as many times as necessary and must terminate with an optimal polynomial with at most $k-1$ inactive roots. This completes the proof.

Remark 3 Although our primary interest is in globally optimal polynomials, the activity result of Theorem 1 holds for locally optimal polynomials as well.

Remark 4 If $\operatorname{dim} \operatorname{null}\left(D_{L^{I}}(0)\right)=k$ where $D_{L^{I}}$ is given in (22), then applying Corollary 2 with $r=k$ leads to $H \subseteq \mathbb{A}$, so that any perturbation $\Delta \in \mathbb{F}^{k}$ is feasible, where $\mathbb{A}$ and $H$ are defined in (7) and (17), respectively. Thus, in this case, there exists an optimizer such that all roots are active.

Remark 5 Though we perturbed a particular subset of $k$ coefficients of $q^{I}$, we could have perturbed any $k$ of the coefficients. We chose to perturb the first $k$ for notational ease. In the complex case, another approach is to factor the inactive part into two monic polynomials, one with degree $k$ and the other one with degree $n_{I}-k$, and perturb the coefficients of the polynomial with degree $k$.

\section{Concluding Remarks}

In this paper, we have presented a remarkable structural property of solutions of polynomial root radius optimization problems subject to affine constraints. An equally important polynomial root max function is the root abscissa, defined as the maximum of the real parts of the roots; this arises in stability analysis of continuous-time dynamical systems. Optimization of the root abscissa of a polynomial subject to affine constraints is a more difficult problem to analyze because, in the case of real coefficients, the infimal abscissa value may not be attained by any optimal polynomial. It was shown in [3] that in the single-constraint case, the infimal value may be approximated arbitrarily accurately by a sequence of polynomials with one set of coincident real roots converging to the optimal value and another set of coincident real roots 
diverging to $-\infty$. Because of the difficulties presented by this issue, we did not consider the polynomial root abscissa in this paper, but leave analysis of this function to future work. More generally, it would be interesting to try to extend our results to all polynomial root max functions.

Another natural idea is to consider extensions from polynomial roots to matrix eigenvalues, but this seems difficult. As with polynomial root max functions, a lot is known about variational analysis of spectral max functions such as the spectral radius and spectral abscissa $[10,8]$. However, simple examples indicate that results analogous to Theorem 1 do not hold for affine matrix families. The reason for this is that although the eigenvalues of a matrix are the roots of its characteristic polynomial, a matrix family depending affinely on a set of parameters does not correspond to an affine characteristic polynomial family, except in special cases such as a companion matrix family. For example, let

$$
A_{0}=\left[\begin{array}{cc}
0.5 & 0 \\
0 & -1
\end{array}\right] \quad \text { and } \quad A_{1}=\left[\begin{array}{cc}
-1 & -2 \\
-0.5 & 1
\end{array}\right] .
$$

The unique global minimizer of the spectral radius of $A_{0}+\lambda A_{1}$ over $\lambda \in \mathbb{R}$ has just one active eigenvalue, so even the simplest case described in Section 2 does not extend from polynomial roots to matrix eigenvalues.

The most important question for future work is whether our results could lead to an efficient algorithm for global minimization of polynomial root max functions subject to affine constraints. In the case of a single constraint, the results in [3] guarantee that a minimizer of the form $(z-\gamma)^{n}$ (in the complex case) or $(z-\gamma)^{m}(z+\gamma)^{n-m}$ (in the real case) exists, and combining this knowledge with the given constraint leads immediately to efficient computation of $\gamma$ (and $m$, in the real case). However, when there is more than one constraint, we have no such formula for an optimal solution. Indeed, it is quite possible that global polynomial root max function optimization with several constraints is hard in a theoretical sense, as is known to be the case for a number of related problems in control $[4,5]$.

\section{References}

1. B. R. Barmish. New tools for robustness of linear systems. Macmilllan, New York, 1993.

2. V. Blondel, M. Gevers, and A. Lindquist. Survey on the state of systems and control. Eur. J. Control, 1:5-23, 1995.

3. V.D. Blondel, M. Gürbüzbalaban, A. Megretski, and M.L. Overton. Explicit solutions for root optimization of a polynomial family with one affine constraint. IEEE T. Automat. Contr., 57(12):3078-3089, Dec 2012.

4. V.D. Blondel and J.N. Tsitsiklis. NP-hardness of some linear control design problems. SIAM J. Control Optim., 35:2118-2127, 1997.

5. V.D. Blondel and J.N. Tsitsiklis. A survey of computational complexity results in systems and control. Automatica, 36(9):1249-1274, 2000.

6. D.N. Burghes and A. Graham. Control and optimal control theories with applications. Elsevier, 2004. 
7. J.V. Burke and J. Eaton. On the subdifferential regularity of max root functions for polynomials. Nonlinear Anal.-Theor., 75(3):1168-1187, Feb 2012.

8. J.V. Burke, A.S. Lewis, and M.L. Overton. Optimal stability and eigenvalue multiplicity. Found. Comput. Math., 1(2):205-225, 2001.

9. J.V. Burke, A.S. Lewis, and M.L. Overton. Variational analysis of functions of the roots of polynomials. Math. Program., 104:263-292, 2005.

10. J.V. Burke and M.L. Overton. Variational analysis of non-Lipschitz spectral functions. Math. Program., 90(2, Ser. A):317-351, 2001.

11. R. Chen. On the problem of direct output feedback stabilization. Proceedings of MTNS, pages 412-414, 1979.

12. R. Chen. Output feedback stabilization of linear systems. PhD thesis, University of Florida, Gainsville, 1979

13. B. Dumitrescu and R. Niemistö. Multistage IIR filter design using convex stability domains defined by positive realness. IEEE T. Signal Proces., 52(4):962-974, Apr 2004

14. A.T. Fam and J.S. Meditch. A canonical parameter space for linear systems design. IEEE T Automat. Contr., 23(3):454-458, 1978.

15. K. Fritzsche and H. Grauert. From Holomorphic Functions to Complex Manifolds. Graduate Texts in Mathematics. Springer, New York, 2002.

16. D. Henrion, M. Sebek, and V. Kucera. Positive polynomials and robust stabilization with fixed-order controllers. IEEE T. Automat. Contr., 48(7):1178-1186, 2003.

17. D. Hinrichsen and A.J. Pritchard. Mathematical systems theory I: modelling, state space analysis, stability and robustness, volume 1. Springer, 2005.

18. A.S. Lewis and M.L. Overton. Nonsmooth optimization via quasi-Newton methods. Math. Program., 141:135-163, 2013.

19. MathWorks Inc. MATLAB, 2014. www.mathworks.com/products/matlab/.

20. J. Moro, J.V. Burke, and M.L. Overton. On the Lidskii-Vishik-Lyusternik perturbation theory for eigenvalues of matrices with arbitrary Jordan structure. SIAM J. Matrix Anal. Appl., 18(4):793-817, 1997.

21. J. Nocedal and S.J. Wright. Numerical Optimization. Springer, 2nd edition, 2000.

22. M.L. Overton. Stability optimization for polynomials and matrices. In O. Kirillov and D. Pelinovsky, editors, Nonlinear Physical Systems: Spectral Analysis, Stability and Bifurcations, pages 351-375. John Wiley and Sons, Inc., 2014.

23. A. Rantzer. Equivalence between stability of partial realizations and feedback stabilization-applications to reduced order stabilization. Linear Algebra Appl., 122:641-653, 1989.

24. Y. Tong and N.K. Sinha. A computational technique for the robust root locus. IEEE T. Ind. Electron., 41(1):79-85, 1994. 